\title{
Evidências sobre a prática mental de tarefas na reabilitação da extremidade superior após acidente vascular encefálico: uma revisão sistemática
}

\author{
Danielle Brandalize ${ }^{\mathrm{a}}$, Michelle Brandalize ${ }^{\mathrm{b}}$ \\ aUniversidade Estadual do Centro-Oeste do Paraná - UNICENTRO, Paraná, PR, Brasil.

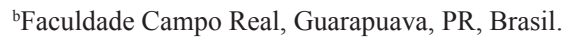

\begin{abstract}
Resumo: Introdução: Apesar de muitos estudos já terem documentado os benefícios da prática mental de tarefas na reabilitação neurológica, ainda não há consenso sobre o melhor protocolo de intervenção. Objetivo: Analisar, através de uma revisão sistemática, o efeito da imaginação motora na reabilitação do membro superior de indivíduos com hemiparesia após acidente vascular encefálico, assim como analisar a eficácia dos protocolos empregados. Metodologia: Foram utilizadas as bases de dados eletrônicas PubMed/Medline, Lilacs e Scielo para busca de artigos publicados no período de 2010 a 2014 sobre o efeito da imaginação motora na recuperação da função da extremidade superior. Resultados: Cinco estudos clínicos randomizados e controlados foram selecionados para este estudo. Os protocolos de intervenção tiveram uma grande variabilidade, sendo que a maioria dos estudos sugere que a prática mental combinada com terapia convencional melhora a função do membro superior de hemiparéticos. Conclusão: A prática mental é uma técnica promissora na recuperação da função após o AVE; no entanto, existem ainda muitas lacunas acerca do protocolo de intervenção. Não há um consenso sobre tempo, intensidade e frequência de intervenção, assim como não está definida qual é a melhor forma de auxiliar a prática mental e em qual fase da reabilitação esta é mais eficaz.
\end{abstract}

Palavras-chave: Acidente Vascular Cerebral, Reabilitação, Extremidade Superior, Atividade Motora.

\section{Evidence on mental task practice in post-stroke upper-limb rehabilitation: a systematic review}

\begin{abstract}
Introduction: Although many studies have already documented the benefits of mental task practice in neurological rehabilitation, the best intervention protocol is still to be defined. Aim: to analyze the effect of motor imagination in upper limb rehabilitation of individuals with hemiparesis after stroke, as well as analyze the effectiveness of the protocols used through a systematic review. Methodology: The electronic databases PubMed / Medline, Lilacs and Scielo were used for searching articles published from 2010 to 2014, on the effect of motor imagination in motor recovery of upper extremity function. Results: Five randomized controlled trials were selected for this study. The intervention protocols have great variability; most studies have suggested that mental task practice combined with conventional therapy improves the hemiparetic upper limb function. Conclusion: The mental practice is a promising technique for recovery after stroke; however there are still many gaps about the intervention protocol. There is no consensus about intervention time, intensity and frequency. As it is unclear what is the best way to help the mental task practice and in which phase of rehabilitation it is mosts effective.
\end{abstract}

Keywords: Stroke, Rehabilitation, Upper Extremity, Motor Activity. 


\section{Introdução}

Uma em cada dez mortes, no mundo, é causada por acidente vascular encefálico (AVE), sendo este a terceira causa de morte nos países desenvolvidos, ficando atrás apenas das doenças coronarianas e do câncer. Mundialmente, 15 milhóes de pessoas sofrem um AVE a cada ano; destas, um terço morre e um terço sobrevive com permanente incapacidade motora (WORLD..., 2004).

Dentre as limitaçóes residuais apresentadas, o membro superior sofre alterações sensoriomotoras evidentes, que podem afetar componentes de desempenho ocupacional, limitando a autonomia do indivíduo para realizar suas atividades de vida diária e no trabalho, e interferindo na sua capacidade de manter uma vida social ativa (AMERICAN..., 2008).

A reabilitação deve ser iniciada o mais breve possível, para ativar o processo de recuperação e reorganização neural (KWAKKEL; KOLLEN; TWISK, 2006). Atualmente, uma vasta gama de modalidades terapêuticas adicionais às técnicas convencionais de reabilitação, está disponível para a reabilitação pós-AVE, tais como: realidade virtual (SARDI; SCHUSTER; ALVARENGA, 2012; POMPEU et al., 2014); terapia por contensão induzida (GROTTA et al., 2004; RIBERTO et al., 2005); terapia orientada a tarefa (PREISSNER, 2010), e prática mental de tarefas. Esta última é uma terapia relativamente nova, que está recebendo muita atenção dentro da pesquisa em reabilitação (BRAUN et al., 2006; CALAYAN; DIZON, 2009; EL-SHENNAWY; EL-WISHY, 2012).

A prática mental foi inicialmente desenvolvida para aperfeiçoar a performance atlética, a fim de melhorar a execução de movimentos em atletas individuais e ajudar na aquisição de novos comportamentos motores (DRISKELL; COPPER; MORAN, 1994), sendo posteriormente adaptada aos programas de reabilitação neurológica, para ajudar na recuperação motora de indivíduos pós-AVE (ZIMMERMANN-SCHLATTER et al., 2008).

Prática mental de tarefas é um método de treinamento durante o qual a pessoa, cognitivamente, ensaia uma habilidade motora usando a imaginação motora, na ausência de movimentos físicos visíveis, com a finalidade de melhorar o desempenho destas habilidades (JACKSON et al., 2001).

Durante a prática mental de tarefas, ocorrem mudanças nas funçóes vegetativas, como frequências cardíaca e respiratória (ROURE et al., 1998) e na atividade muscular (GUILLOT et al., 2007) de forma semelhante às observadas durante a prática física. Possivelmente, a linha mais convincente vem de estudos que investigaram as relaçôes neurais da imaginação e da prática física, e que mostram evidências de que, durante sessóes de prática mental, praticamente as mesmas áreas cerebrais são ativadas quando comparadas com as áreas cerebrais ativadas durante a execução física de tarefas funcionais (PAGE et al., 2009). Ietswaart et al. (2006), com base nessas observaçóes, sugerem que imaginar os movimentos da mão poderia estimular a redistribuição de atividade cerebral que acompanha recuperação da função da mão, resultando na redução do déficit motor pós-AVE.

A prática mental de tarefas é uma técnica de baixo custo e fácil acesso, pois pode ser aplicada com segurança e dispensa instalações especiais, tornando-se apropriada para ser inserida no cotidiano da Fisioterapia e da Terapia Ocupacional (DIJKERMAN et al., 2004); além disso, pode ser praticada em todas as fases da reabilitaçáo (JACKSON et al., 2001).

Devido aos resultados positivos da prática mental obtidos no esporte (DRISKELL; COPPER; MORAN, 1994), muitos pesquisadores tiveram interesse em avaliar seu efeito na reabilitação neurológia (JACKSON et al., 2001; DIJKERMAN et al., 2004; PAGE et al., 2005). Algumas revisôes sistemáticas foram feitas sobre o tema: uma sobre a prática mental de tarefas na reabilitaçáo de indivíduos com Doença de Parkinson e AVE (BRAUN et al., 2013); quatro sobre o efeito da prática mental de tarefas de forma geral na reabilitaçáo do AVE, sem focar na extremidade superior (BRAUN et al., 2006; ZIMMERMANN-SCHLATTER et al., 2008; CALAYAN; DIZON, 2009; EL-SHENNAWY; EL-WISHY, 2012) e duas com foco na extremidade superior; uma com publicações até 2005 (SHARMA et al., 2006), e outra até 2009 (NILSEN et al., 2010). Embora essas duas últimas revisóes tenham apresentado consideraçóes positivas em relação aos benefícios da prática mental de tarefas na reabilitação da extremidade superior após AVE, ambas concluíram que ainda falta um consenso em relação ao protocolo de tratamento utilizado.

Considerando-se a relevância de investigar os efeitos da prática mental de tarefas na reabilitação neurológica e a necessidade da formação de um consenso a respeito de um protocolo de tratamento eficiente, o objetivo do presente estudo foi analisar as evidências mais atuais sobre o efeito da prática mental de tarefas, especificamente na reabilitação da extremidade superior de indivíduos hemiparéticos após AVE, assim como analisar a eficácia dos protocolos empregados. 


\section{Metodologia}

Trata-se de uma revisão sistemática da literatura acerca do uso da prática mental de tarefas na reabilitação neurológica, para formar conclusôes baseadas em conhecimentos produzidos por pesquisas primárias sobre o assunto. A busca e a seleção dos artigos foram conduzidas conforme consta na Figura 1.

\subsection{Busca de artigos}

Duas pesquisadoras independentes conduziram uma busca nas bases de dados PubMed/ Medline, Scielo e Lilacs, com os seguintes filtros: ensaio clínico aleatório (ECA) e período de publicação, os últimos cinco anos. As buscas foram realizadas de setembro a outubro de 2014.

\section{Objetivo}

1) Analisar as evidências sobre o efeito da prática mental de tarefas na reabilitação do membro superior após AVE 2) Identificar a variação dos protocolos de prática mental utilizados e suas implicaçóes nos resultados.

\begin{tabular}{|l|l|}
\multicolumn{1}{c|}{$\begin{array}{c}\text { Critérios de seleçáo } \\
\text { - ECR publicados em inglês, no período } \\
\text { de janeiro de 2009 a outubro de 2014. } \\
\text { - Indivíduos com sequelas de AVE } \\
\text { - Intervenção: prática mental com } \\
\text { imaginaçáo motora } \\
\text { - Desfechos relacionados à função motora } \\
\text { do membro superior }\end{array}$} \\
\hline
\end{tabular}

\section{Artigos identificados na busca aos \\ bancos de dados $(\mathbf{n}=27)$ \\ Pubmed/Medline $(n=24)$ \\ Scielo e Lilacs $(\mathrm{n}=3)$}

\section{Exclusáo de artigos leitura de títulos e resumos}

(1) Não apresentarem o desfecho estipulado

(2) Utilizarem equipamentos específicos como espectroscopia ou estimulação eletromiográfica

(3) Apresentarem outra população de estudo

(4) Não apresentarem a prática mental e/ou imaginação motora como intervençấo

6 artigos selecionados

Uma exclusão após a extração dos dados por não investigar, especificamente $o$ efeito da prática mental.

5 artigos foram incluídos no estudo:

- Riccio et al., 2010

- Page et al., 2011

- Letswaart et al., 2011

- Nilsen et al., 2012

- Timmermans et al., 2013

Figura 1. Fluxograma do processo de seleção de artigos. 
Os descritores utilizados foram adotados mediante consulta ao DeCS (Descritores de Ciências da Saúde) da BVS (Biblioteca Virtual em Saúde), sendo eleitas as combinações de termos na língua inglesa: 1. Stroke AND mental practice AND upper limb; 2. Stroke AND mental practice AND arm; 3. Stroke $A N D$ motor imagery $A N D$ upper limb; 4. Stroke AND motor imagery $A N D$ arm.

\subsection{Critérios de seleção dos artigos}

\subsubsection{Tipos de estudos}

Foram considerados apenas estudos com nível de evidência I, ou seja, ensaios clínicos randomizados e controlados (BRAUN et al., 2006; NILSEN et al., 2010), publicados nos últimos cinco anos (2010-2014), nos idiomas português, inglês e espanhol. Foram selecionados, para a presente revisão, estudos que investigaram o efeito da prática mental de tarefas, associada ou não a outro tipo de terapia para a recuperação da função da extremidade superior em indivíduos com hemiparesia pós-AVE. Os estudos deviam apresentar desfechos que estivessem relacionados à função motora do membro superior acometido, como escalas de funcionalidade, medidas de força e destreza.

\subsection{Avaliação e síntese dos artigos}

Os estudos selecionados foram avaliados pela qualidade metodológica em conformidade com a Escala de PEDro (MAHER et al., 2003; SAMPAIO; MANCINI, 2007). A Escala de PEDro é uma escala de 11 itens, projetada para avaliação da qualidade metodológica de ensaios controlados randomizados. Cada item satisfeito (exceto o item 1, que, ao contrário de outros itens na escala, diz respeito à validade externa) contribui com um ponto para a pontuação total de PEDro, que tem um intervalo de 0 a 10 pontos (SAMPAIO; MANCINI, 2007), sendo que escores $\geq 5$ são considerados de alta qualidade metodológica (MOSELEY et al., 2002). A pontuação total de cada estudo selecionado para esta revisão, assim como a pontuaçáo obtida em cada item para cada estudo, separadamente, estáo apresentadas na Tabela 1 .

Os resultados e metodologias dos estudos estão apresentados na Tabela 2. Em razáo da variedade de metodologias empregadas nos artigos, não foi

Tabela 1. Estudos inclusos na análise e qualidade metodológica pela escala PEDro.

\begin{tabular}{lcccccc}
\hline \multicolumn{1}{c}{ Validade interna } & $\begin{array}{c}\text { Riccio et al. } \\
(\mathbf{2 0 1 0})\end{array}$ & $\begin{array}{c}\text { Page et al. } \\
(\mathbf{2 0 1 1})\end{array}$ & $\begin{array}{c}\text { Ietswaart et al. } \\
\mathbf{( 2 0 1 1 )}\end{array}$ & $\begin{array}{c}\text { Nilsen et al. } \\
\mathbf{( 2 0 1 2 )}\end{array}$ & $\begin{array}{c}\text { Timmermans } \\
\text { et al. (2013) }\end{array}$ \\
\hline Critérios de elegibilidade & - & - & - & - & - \\
\hline Alocação aleatória & 1 & 1 & 1 & 1 & 1 \\
\hline Sigilo na alocação & 1 & 1 & 1 & 1 & 1 \\
\hline $\begin{array}{l}\text { Similaridade dos grupos na fase } \\
\text { inicial }\end{array}$ & 1 & 1 & 1 & 1 & 1 \\
\hline Cegamento dos sujeitos & 0 & 0 & 0 & 0 & 0 \\
\hline Cegamento dos terapeutas & 0 & 1 & 0 & 0 & 0 \\
\hline Cegamento dos avaliadores & 1 & 1 & 1 & 1 & 1 \\
\hline $\begin{array}{l}\text { Medida de pelo menos um } \\
\text { desfecho primário } \\
\text { em 85\% dos sujeitos alocados }\end{array}$ & 1 & 1 & 1 & 1 & 1 \\
\hline $\begin{array}{l}\text { Análise de intenção de } \\
\text { tratamento }\end{array}$ & 0 & 0 & 1 & 0 & 1 \\
\hline $\begin{array}{l}\text { Comparação entre grupos de } \\
\text { pelo menos um desfecho } \\
\text { primário }\end{array}$ & 1 & 1 & 1 & 1 & 1 \\
\hline
\end{tabular}

Relato de medidas de variabilidade e estimativa dos parâmetros de pelo menos uma variável primária

\begin{tabular}{lccccc}
\hline Total & 7 & 8 & 9 & 7 & 8 \\
\hline Cada item recebe uma avaliação de 0 a 1. O escore total na Escala de PEDro é igual a 10, sendo que escores iguais ou maiores
\end{tabular}
do que 5 apresentam alta qualidade metodológica. 


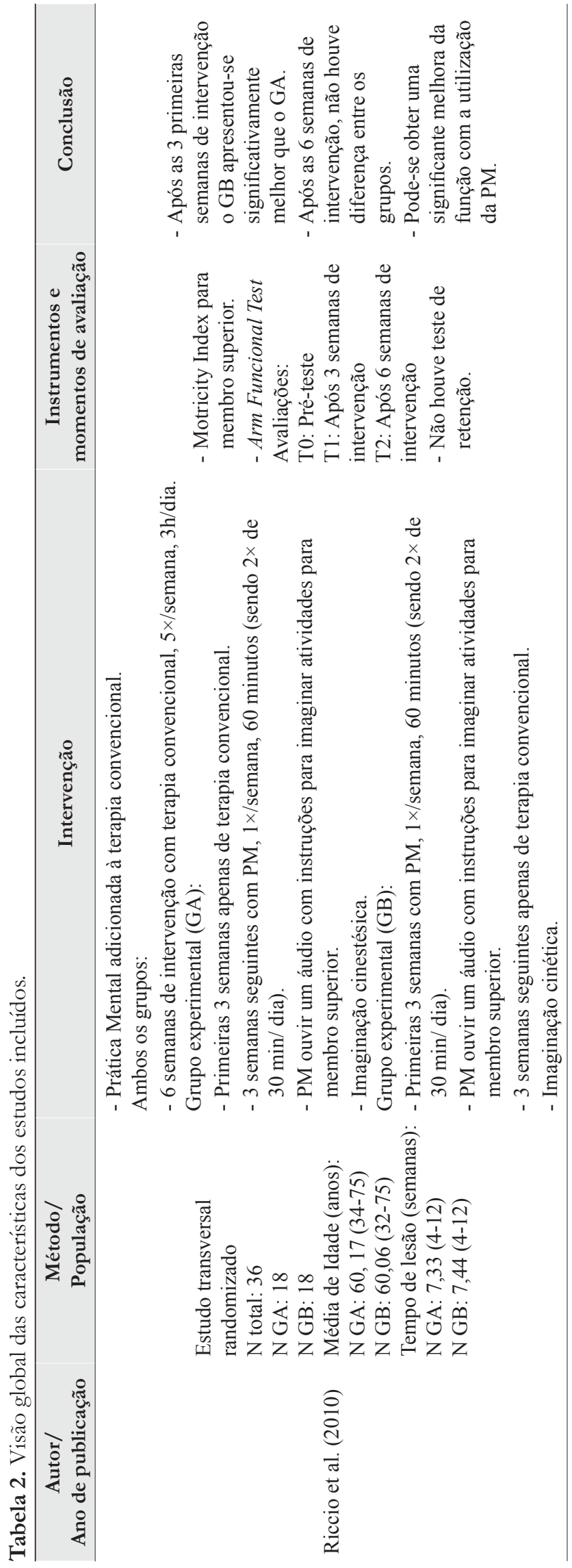




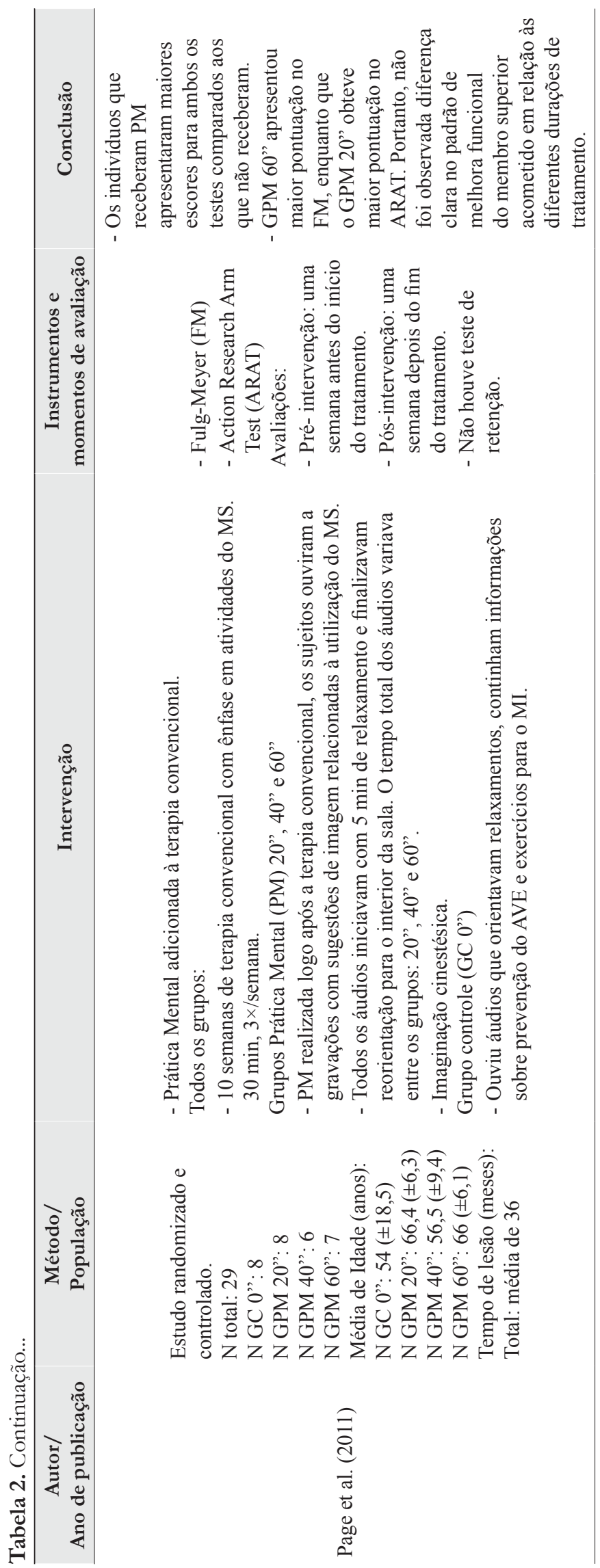




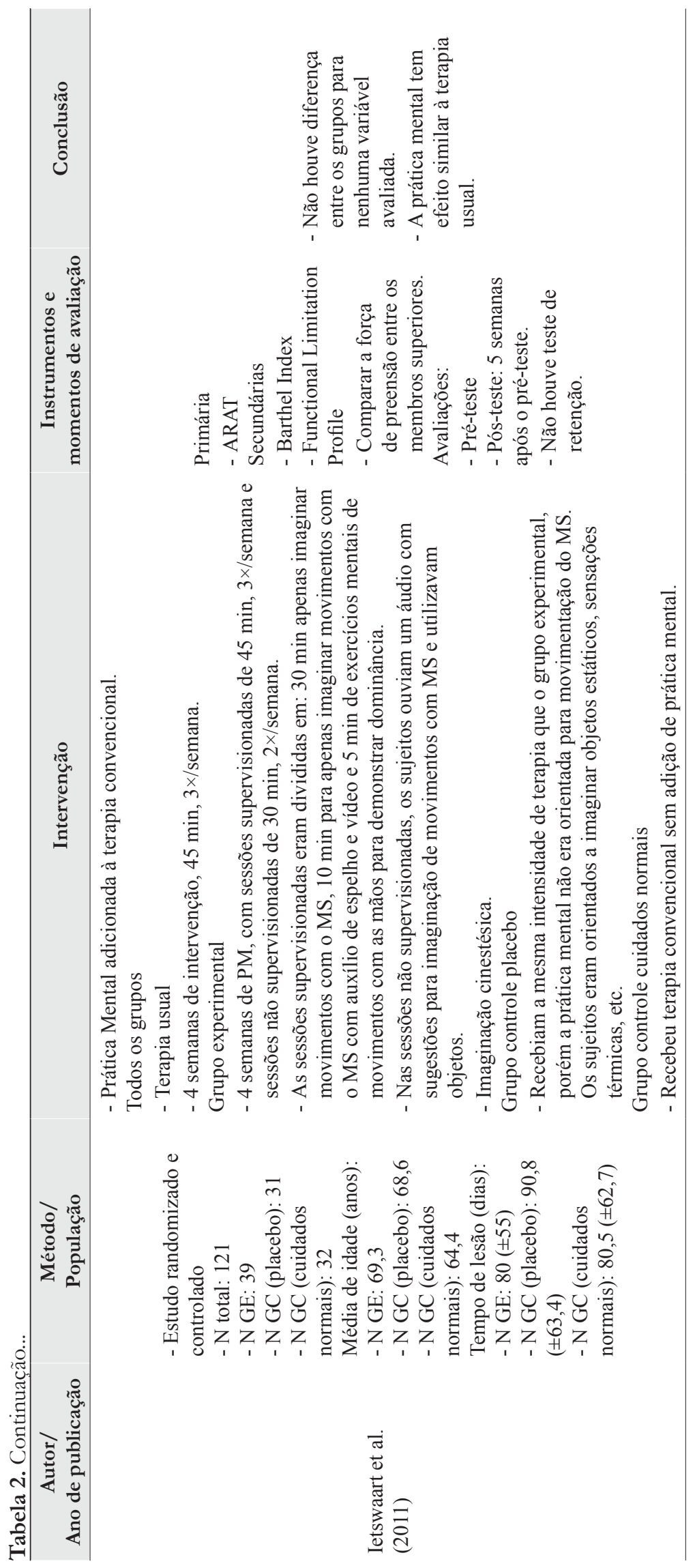




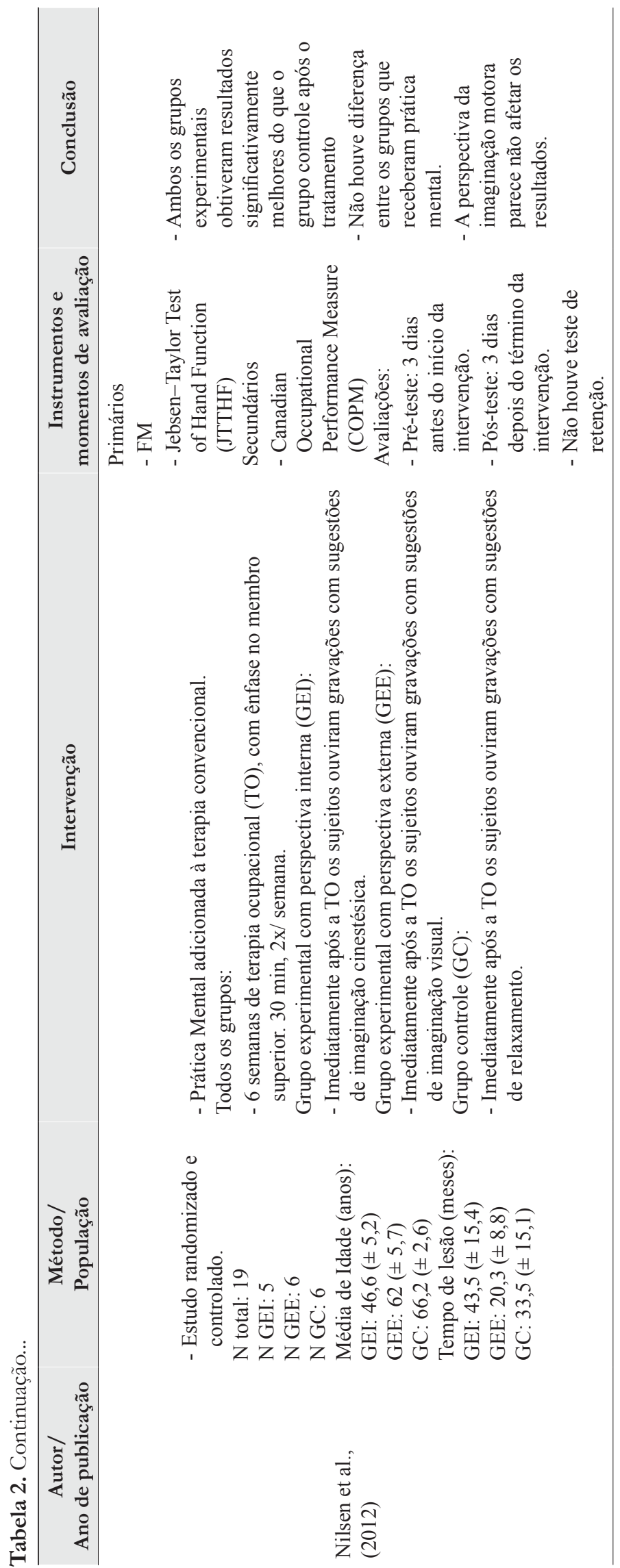




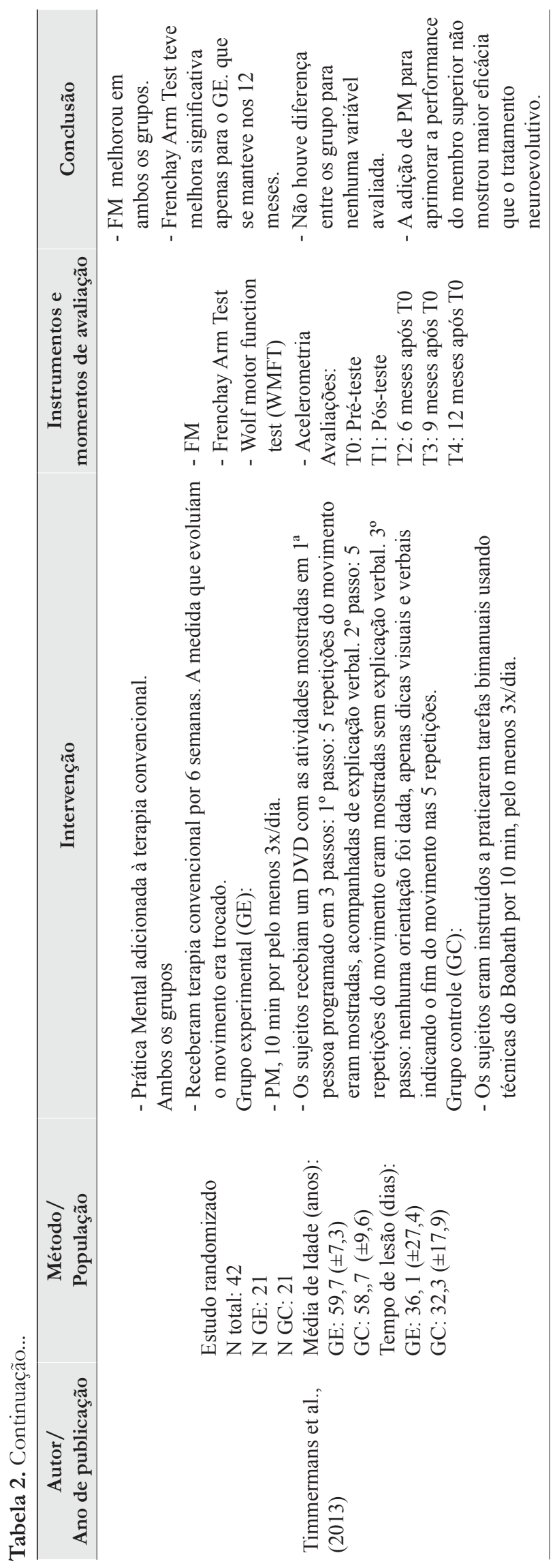


desenvolvida análise estatística dos dados. Efetuou-se uma comparação direta dos resultados entre os grupos analisados.

\section{Resultados}

Um total de 27 artigos foi listado nas bases de dados Pubmed/Medline ( $\mathrm{n}=24)$ e Scielo/Lilacs ( $\mathrm{n}=3$ ), para a leitura de títulos e resumos. Após tal leitura, foram selecionados seis estudos potencialmente capazes de responder as perguntas desta revisão. Os demais artigos foram excluídos por (1) não apresentarem o desfecho estipulado; (2) utilizarem equipamentos específicos como espectroscopia ou estimulação eletromiográfica; (3) apresentarem outra população de estudo, e (4) não apresentarem a prática mental e/ou imaginação motora como intervenção. Após a extração dos dados, um artigo foi excluído, pois apesar de incluir prática mental de tarefas em seu protocolo de intervenção, não investigou especificamente seu efeito. Totalizaram-se, dessa forma, cinco artigos inclusos nesta revisão (Figura 1).

Todos os estudos selecionados apresentaram escore $\geq 5$ na escala PEDro, sendo considerados, portanto, de alta qualidade metodológica (Tabela 1); os escores da validade interna variaram de 7 a 9 . Todos os ECAs foram classificados positivamente para alocaçáo aleatória dos grupos, similaridade entre os grupos, cegamento dos avaliadores, medida de pelo menos um desfecho primário em $85 \%$ dos sujeitos alocados e comparação entre grupos de pelo menos um desfecho primário. Um estudo foi positivo para cegamento dos participantes e um para cegamento dos terapeutas, e dois estudos apresentaram análise de intenção de tratamento. As características dos artigos inclusos quanto a populaçáo, método, intervençáo, desfecho e resultado estão apresentadas na Tabela 2, para permitir a comparação entre estes.

\subsection{Efeitos em relação às características dos participantes}

Participaram dos estudos, sujeitos de ambos os gêneros, sendo a média de idade dos participantes de todos os estudos similar, variando de 46,6 a 69,3 anos. Todos os estudos incluíram indivíduos com hemiparesia direita e esquerda. O tempo de lesão foi consideravelmente diferente entre os estudos, variando de 32,2 dias a 43,5 meses, sendo que três estudos avaliaram sujeitos na fase subaguda do AVE (RICCIO et al., 2010; IETSWAART et al., 2011; TIMMERMANS et al., 2013) e dois investigaram sujeitos na fase crônica de recuperação do AVE (PAGE et al., 2011; NILSEN et al., 2012).

\subsection{Efeitos sobre a função do membro superior}

Dos cinco artigos selecionados, três mostraram resultados positivos da prática mental para melhorar a funcionalidade do membro superior de hemiparéticos após AVE (RICCIO et al., 2010; PAGE et al., 2011; NILSEN et al., 2012). Enquanto um encontrou resultados positivos para ambos os grupos, experimental e controle (IETSWAART et al., 2011), e outro, ainda, demonstrou resultados positivos em favor da prática mental, porém não para todas as medidas (TIMMERMANS et al., 2013).

\subsection{Avaliação da função de membro superior}

Todos os estudos utilizaram mais de um instrumento para medir a atividade e/ou a função da extremidade superior. Dentre os instrumentos de medida utilizados como desfecho primário, a Fulg-Meyer (FM) foi a escala mais frequente, presente em três estudos (PAGE et al., 2011; NILSEN et al., 2012; TIMMERMANS et al., 2013). O teste de pesquisa-ação do braço (ARAT - Action Research Arm Test) foi usado em dois dos estudos selecionados (IETSWAART et al., 2011; PAGE et al., 2011); o teste de função motora de Wolf (WMFT - Wolf motor function test) foi utilizado apenas por Timmermans et al. (2013), que também realizaram Frenchay Arm Test e acelerometria. Nilsen et al. (2012), além do FM, foram os únicos a utilizar o Jebsen-Taylor Test of Hand Function (JTTHF).

A maioria dos estudos avaliou o efeito em curto prazo da prática mental de tarefas, sendo que apenas um realizou avaliaçóes de retenção (TIMMERMANS et al., 2013).

\subsection{Efeitos em relação às características da intervenção}

Todos os estudos adicionaram a prática mental na terapia convencional de reabilitação (RICCIO et al., 2010; PAGE et al., 2011; IETSWAART et al., 2011; NILSEN et al., 2012; TIMMERMANS et al., 2013). Três estudos especificaram que as atividades práticas (terapia convencional) eram com ênfase em movimentos e tarefas do membro superior (RICCIO et al., 2010; PAGE et al., 2011; NILSEN et al., 2012); os outros dois estudos não citam detalhes sobre a terapia convencional, Ietswaart et al. (2011) apenas citam que os participantes continuaram fazendo a terapia convencional que faziam com seus próprios terapeutas 
e Timmermans et al. (2013) não detalham mais informaçóes.

A frequência de intervenção variou de três vezes ao dia até duas vezes na semana; a duração de cada sessão teve bastante variação entre os estudos $(20,30$, 40,45 e 60 minutos) e o tempo total de intervençáo variou de quatro (IETSWAART et al., 2011) a dez (PAGE et al., 2011) semanas, sendo que, dos cinco estudos inclusos nesta revisão, três tiveram intervenção com duração de seis semanas (RICCIO et al., 2010; IETSWAART et al., 2011; NILSEN et al., 2012). Em relação ao tempo de cada sessão, um estudo comparou a diferença entre 20,40 e 60 minutos, mas não observaram um padrão claro da melhora na função e a duração de tratamento (PAGE et al., 2011).

Em relação ao tipo de prática mental, a maioria dos estudos orientou a prática mental de tarefas de uma perspectiva cinética ou interna (RICCIO et al., 2010; PAGE et al., 2011; IETSWAART et al., 2011; TIMMERMANS et al., 2013); um estudo utilizou ambas as perspectivas de prática mental, interna e externa, e comparou o resultado entre os grupos, não encontrando diferença entre estas (NILSEN et al., 2012). Quatro estudos (RICCIO et al., 2010; PAGE et al., 2011; NILSEN et al., 2012; TIMMERMANS et al., 2013) realizaram a prática mental imediatamente após a terapia convencional e um (IETSWAART et al., 2011) realizou a sessão de prática mental, separadamente da terapia convencional.

A principal estratégia utilizada para a intervenção da prática mental foi o áudio tape (RICCIO et al., 2010; PAGE et al., 2011; NILSEN et al., 2012); um estudo utilizou DVD (TIMMERMANS et al., 2013) e outro associou áudio tape com espelhos e objetos, além de intercalar sessóes supervisionadas e não supervisionadas por um terapeuta (IETSWAART et al., 2011). As tarefas utilizadas durante as intervençôes variaram: as tarefas do teste funcional para membro superior (Arm functional test) (RICCIO et al., 2010); alcançar e apertar uma caneca ou objeto, virar a página de um livro, usar apropriadamente um utensílio de escrita e outro de alimentação, e usar uma escova de cabelo ou pente (PAGE et al., 2011); abrir e fechar a mão, rotação de punho e elevaçáo do braço, alcançar/pegar/soltar utensílios domésticos, passar roupa e lavar embaixo dos braços ou abotoar uma camisa (IETSWAART et al., 2011); beber de uma caneca, desabotoar um botáo (vestindo uma camisa) e dobrar uma toalha (NILSEN et al., 2012); segurar papel, apertar/segurar/soltar um copo, segurar uma caneta para marcar um X no papel, atender uma ligação telefônica, trazer um copo à boca e colocar água de uma jarra em um copo (TIMMERMANS et al., 2013).

\section{Discussão}

Esta revisão teve como objetivo encontrar evidências sobre a recuperação da função do membro superior após um AVE, utilizando-se a prática mental de tarefas no programa de reabilitação. Há evidências de que a prática mental de tarefas associada à terapia convencional demonstrou ser eficaz para aumentar a funcionalidade do membro superior de indivíduos com hemiparesia (RICCIO et al., 2010; PAGE et al., 2011; NILSEN et al., 2012). Contudo, foram identificados poucos estudos sobre o tema, nos quais os protocolos de intervenção de prática mental variaram muito.

Clinicamente, os resultados indicam que combinar a prática mental de tarefas à terapia convencional traz mais benefícios do que aplicar a terapia convencional isoladamente; contudo, a adição da prática mental não mostrou benefícios quando esta foi realizada separadamente da prática física (IETSWAART et al., 2011).

A prática mental de tarefas pode ser utilizada em todas as fases de recuperação do AVE (JACKSON et al., 2001). Numa fase inicial, essa terapia permite que os pacientes pratiquem mentalmente uma tarefa que ainda náo podem realizar fisicamente devido ao prejuízo motor. Embora existam evidências de que a reabilitação deva começar o mais rapidamente possível (BIERNASKIE; CHERNENKO; CORBETT, 2004; KWAKKEL; KOLLEN; TWISK, 2006), ainda não está estabelecido quando é melhor iniciar com essa terapia (ZIMMERMANN-SCHLATTER et al., 2008).

O estudo de Riccio et al. (2010) encontrou resultados positivos para a recuperação da função do membro superior com a prática mental em indivíduos na fase subaguda do AVE; porém, dois estudos com participantes, também na fase subaguda de reabilitação (IETSWAART et al., 2011; TIMMERMANS et al., 2013), não observaram superioridade na recuperação com a adição da prática mental de tarefas. Parece que os efeitos positivos dessa terapia são mais evidentes na fase crônica.

Apenas dois dos cinco estudos selecionados preocuparam-se em avaliar diferentes protocolos de intervenção com prática mental de tarefas: um deles investigou o efeito do tempo de intervenção (PAGE et al., 2011) e outro verificou os resultados das perspectivas de imaginação (interna e externa) (NILSEN et al., 2012). Com o estudo de Page et al. (2011), não foi possível definir o melhor tempo de duração de uma sessão de prática mental, pois não houve diferença significativa entre os grupos que realizaram 20, 40 e 60 minutos. E, embora publicaçôes 
anteriores (LOREY et al., 2009) tenham defendido que realizar prática mental de uma perspectiva interna traria mais resultados positivos do que de uma perspectiva externa, no estudo controlado e randomizado de Nilsen et al. (2012), uma perspectiva não foi superior à outra.

Os principais vieses relacionados aos estudos inclusos nesta revisão foram cegamento dos terapeutas e participantes; nâo obstante, é importante considerar que o cegamento dos sujeitos e terapeutas é difícil ou quase impraticável devido à participação ativa do participante e à necessidade de instruçôes dadas pelos terapeutas, além da falta de medidas de retenção para avaliar o efeito em longo prazo da prática mental. Apesar de algumas limitaçôes, a média de 7,8 na Escala PEDro representa uma boa qualidade metodológica dos ECAs inclusos na presente revisão.

Limitações desta revisão sistemática incluem a possibilidade de faltarem alguns estudos devido às combinaçóes dos termos de busca e à terminologia usadas, além da inclusão de estudos publicados apenas nos idiomas inglês, português e espanhol, e de um reduzido número de base de dados. Para as informaçôes que faltavam ou não estavam claras nos estudos, o critério de avaliação de qualidade não recebeu pontuação; no entanto, a informação não reportada não significa, necessariamente, que não foi realizada, podendo haver distorção na avaliação da qualidade de alguns estudos.

Todavia, essa revisáo buscou relacionar dados atuais (últimos cinco anos) sobre um assunto bem específico: a função do membro superior, bem como elucidar os principais pontos dos programas de tratamento utilizados, a fim de nortear os terapeutas interessados em aplicar a prática mental em seus protocolos de reabilitação. Embora ainda haja a necessidade de maior comprovação sobre seus efeitos, pode-se observar que, para a prática clínica, os seguintes fatores sobre a prática mental devem ser considerados: a prática mental associada à prática física e a sua aplicação na fase crônica de recuperação apresentaram melhores resultados, não havendo diferença em relação às perspectivas internas e externas.

Em suma, não há consenso entre os estudos sobre protocolo da intervenção. As evidências nesta área ainda precisam ser determinadas por mais ECAs de alta qualidade metodológica. Investigaçôes futuras devem determinar a melhor frequência, a intensidade, a duração, a perspectiva de imaginação e os métodos utilizados para facilitar a imaginação. É necessário avaliar a eficácia da prática mental realizada separadamente da prática física. A grande variação no tamanho da amostra encontrada nos estudos pode dificultar a generalizaçáo dos resultados; dessa forma, estudos futuros precisam ter uma amostra grande o suficiente para possibilitar a divisão em subgrupos e determinar, com maior precisão, a fase de reabilitação e quais características dos participantes são imprescindíveis para se obterem os benefícios da prática mental de tarefas.

\section{Considerações finais}

Dos cinco estudos avaliados, três apontaram resultados positivos com a associação da prática mental de tarefas à prática física. No entanto, a grande variabilidade dos resultados dificulta a generalização dos resultados.

\section{Referências}

AMERICAN OCCUPATIONAL THERAPY ASSOCIATION - AOTA. Occupational therapy practice framework: domain and process. American Journal of Occupational Therapy, New York, v. 62, n. 6, p. 625-683, 2008. http://dx.doi.org/10.5014/ajot.62.6.625.

BIERNASKIE, J.; CHERNENKO, G.; CORBETT, D. Efficacy of rehabilitative experience declines with time after focal ischemic brain injury. The Journal of Neuroscience: The Official Journal of the Society for Neuroscience, Washington, v. 24, n. 5, p. 1245-1254, 2004. http:// dx.doi.org/10.1523/JNEUROSCI.3834-03.2004. PMid:14762143.

BRAUN, S. M. et al. The effects of mental practice in stroke rehabilitation: a systematic review. Archives of Physical Medicine and Rehabilitation, Reston, v. 87, n. 6, p. 842-852, 2006. http://dx.doi.org/10.1016/j. apmr.2006.02.034. PMid:16731221.

BRAUN, S. et al. The effects of mental practice in neurological rehabilitation: a systematic review and meta-analysis. Frontiers in Human Neuroscience, Lausanne, v. 7, p. 1-23, 2013. PMid:23935572.

CALAYAN, S. L. M.; DIZON, J. M. R. A systematic review on the effectiveness of mental practice with motor imaginary in the neurologic rehabilitation on stroke patients. Journal of Allied Health Science and Practice, Florida, v. 7, n. 2, p. 1-11, 2009.

DIJKERMAN, H. C. et al. Does motor imagery training improve hand function in chronic stroke patients? A pilot study. Clinical Rehabilitation, London, v. 18, n. 5, p. 538-549, 2004. http://dx.doi. org/10.1191/0269215504cr769oa. PMid:15293488.

DRISKELL, J. E.; COPPER, C.; MORAN, A. Does mental practice enhance performance? Journal of Applied Psychology, Washington, v. 79, n. 4, p. 481-492, 1994. http://dx.doi.org/10.1037/0021-9010.79.4.481. 
EL-SHENNAWY, S. A. W.; EL-WISHY, A. A. A systematic review of efficacy of mental practice in chronic stroke rehabilitation. Egypt Journal of Neurology Neurosurgery \& Psychiatry, Cairo, v. 49, n. 3, p. 173-180, 2012.

GUILLOT, A. et al. Muscular responses during motor imagery as a function of muscle contraction types. International Journal of Psychophysiology, Netherlands, v. 66, n. 1, p. 18-27, 2007. http://dx.doi.org/10.1016/j. ijpsycho.2007.05.009.

GROTTA, J. C. et al. Constraint-induced movement therapy. Stroke, Dallas, v. 35, n SE1, p. 2699-2701, 2004. Suplemento 1. http://dx.doi.org/10.1161/01. STR.0000143320.64953.c4

IETSWAART, M. et al. Recovery of hand function through mental practice: a study protocol. BMC Neuro$\log y$, London, v. 6, p. 1-7, 2006. PMid:17067370.

IETSWAART, M. et al. Mental practice with motor imagery in stroke recovery: randomized controlled trial of efficacy. Brain, London, v. 134, n. Pt 5, p. 13731386, 2011. http://dx.doi.org/10.1093/brain/awr077. PMid:21515905.

JACKSON, P. L. et al. Potential role of mental practice using motor imagery in neurologic rehabilitation. Archives of Physical Medicine and Rehabilitation, Reston, v. 82, n. 8, p. 1133-1141, 2001. http://dx.doi.org/10.1053/ apmr.2001.24286. PMid:11494195.

KWAKKEL, G.; KOLLEN, B.; TWISK, J. Impact of time on improvement of outcome after stroke. Stroke, Dallas, v. 37, n. 9, p. 2348-2353, 2006. http:// dx.doi.org/10.1161/01.STR.0000238594.91938.1e. PMid:16931787.

LOREY, B. et al. The embodied nature of motor imagery: the influence of posture and perspective. Experimental Brain Research, London, v. 194, n. 2, p. 233-243, 2009. http://dx.doi.org/10.1007/s00221-008-1693-1. PMid:19139856.

MAHER, C. G. et al. Reliability of the PEDro scale for rating quality of randomized controlled trials. Physical Therapy, Alexandria, v. 83, n. 8, p. 713-721, 2003. PMid:12882612.

MOSELEY, A. M. et al. Evidence for physiotherapy practice: a survey of the physiotherapy evidence database (PEDro). Aust Journal of Physiotherapy, Australia, v. 48, n. 1, p. 43-49, 2002. http://dx.doi.org/10.1016/S00049514(14)60281-6. PMid:11869164.

NILSEN, D. M.; GILLEN, G.; GORDON, A. M. Use of mental practice to improve upper-limb recovery after stroke: a systematic review. American Journal of Occupational Therapy, New York, v. 64, n. 5, p. 695708, 2010. http://dx.doi.org/10.5014/ajot.2010.09034. PMid:21073100.

NILSEN, D. M. et al. Effect of Imagery Perspective on occupational performance after stroke: a randomized controlled trial. American Journal of Occupational Thera- py, New York, v. 66, n. 3, p. 320-329, 2012. http://dx.doi. org/10.5014/ajot.2012.003475. PMid:22549597.

PAGE, S. J.; LEVINE, P.; LEONARD, A. C. Effects of mental practice on affected limb use and function in chronic stroke. Archives of Physical Medicine and Rehabilitation, Reston, v. 86, n. 3, p. 399-402, 2005. http://dx.doi.org/10.1016/j.apmr.2004.10.002. PMid:15759218.

PAGE, S.J. et al. Cortical plasticity following motor skill learning during mental practice in stroke. Neurorehabilitation Neural Repair, Thousand Oaks, v. 23, n. 4, p. 382-388, 2009. http://dx.doi. org/10.1177/1545968308326427.

PAGE, S. J. et al. Longer versus shorter mental practice sessions for affected upper extremity movement after stroke: A RANDOMIZED controlled trial. Clinical Rehabilitation, London, v. 25, n. 7, p. 627-637, 2011. http://dx.doi.org/10.1177/0269215510395793. PMid:21427151.

PREISSNER, K. Use of the occupational therapy task-oriented approach to optimize the motor performance of a client with cognitive limitations. The American Journal of Occupational Therapy, New York, v. 64, n. 5, p. 727734, 2010. http://dx.doi.org/10.5014/ajot.2010.08026. PMid:21073103.

POMPEU, J. E. et al. Os efeitos da realidade virtual na reabilitação do acidente vascular encefálico: uma revisão sistemática. Motricidade, Ribeira de Pena, v. 10, n. 4, p. 111-122, 2014. http://dx.doi.org/10.6063/motricidade.10(4).3341.

RIBERTO, M. et al. Terapia de restrição como forma de aprimoramento da função do membro superior em pacientes com hemiplegia. Acta Fisiatrica, São Paulo, v. 12, n. 1, p. 15-19, 2005. http://dx.doi.org/10.5935/01047795.20050001 .

RICCIO, I. et al. Mental practice is effective in upper limb recovery after stroke: a randomized single-blind cross-over study. European Journal of Physical and Rehabilitation Medicine, Torino, v. 46, n. 1, p. 19-25, 2010. PMid:20332722.

ROURE, R. et al. Autonomic nervous system responses correlate with mental rehearsal in volleyball training. European Journal of Applied Physiology and Occupational Physiology, Berlin, v. 78, n. 2, p. 99-108, 1998. http:// dx.doi.org/10.1007/s004210050393. PMid:9694307.

SAMPAIO, R. F.; MANCINI, M. C. Estudos de revisão sistemática: um guia para síntese criteriosa da evidência científica. Revista Brasileira de Fisioterapia, São Carlos, v. 11, n. 1 , p. 83-89, 2007. http://dx.doi.org/10.1590/ S1413-35552007000100013.

SARDI, S. D.; SCHUSTER, R. C.; ALVARENGA, L. F. C. Efeitos da realidade virtual em hemiparéticos crônicos pós-acidente vascular encefálico. Revista Brasileira de Ciências da Saúde, São Caetano do Sul, v. 10, n. 32, p. 29-35, 2012. 
SHARMA, N.; POMEROY, V. M.; BARON, J. C. Motor imagery: a backdoor to the motor system after stroke? Stroke, Dallas, v. 37, n. 7, p. 1941-1952, 2006. http:// dx.doi.org/10.1161/01.STR.0000226902.43357.fc. PMid:16741183.

TIMMERMANS, A. A. A. et al. Effect of mental practice on the improvement of function and daily activity performance of the upper extremity in patients with subacute stroke: a randomized clinical trial. Journal of the American Medical Directors Association, New York, v. 14, n. 3, p. 204-2012, 2013. http://dx.doi.org/10.1016/j. jamda.2012.10.010. PMid:23273853.

WORLD HEALTH ORGANIZATION - WHO. The atlas of heart disease and stroke. Genebra: WHO, 2004.

ZIMMERMANN-SCHLATTER, A. et al. Efficacy of motor imagery in post-stroke rehabilitation: a systematic review. Journal of NeuroEngineering and Rehabilitation, London, v. 5, n. 8, p. 1-10, 2008. Disponível em: <http://www.jneuroengrehab.com/content/5/1/8>. Acesso em: 23 jan. 2015.

\section{Contribuição dos Autores}

Ambas as autoras trabalharam na busca das fontes de dados, na elaboração e na revisão do texto. As autoras aprovaram a versão final do artigo. 